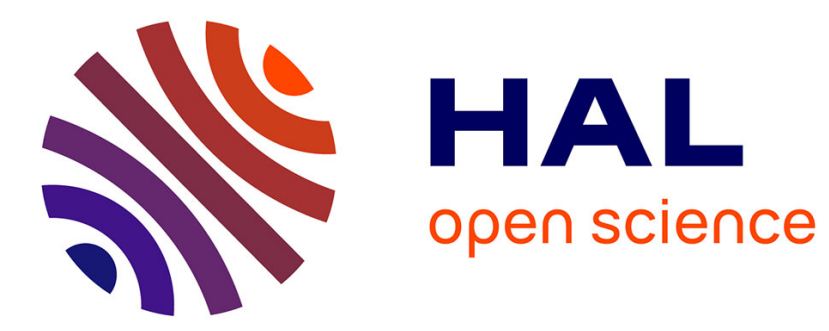

\title{
L'olfaction dans les troubles dépressifs : intérêts et perspectives
}

Gérard Brand, Benoist Schaal

\section{To cite this version:}

Gérard Brand, Benoist Schaal. L'olfaction dans les troubles dépressifs : intérêts et perspectives. L'Encéphale, 2017, 43 (2), pp.176-182. 10.1016/j.encep.2016.04.008 . hal-01564807

\section{HAL Id: hal-01564807 \\ https://u-bourgogne.hal.science/hal-01564807}

Submitted on 6 Oct 2017

HAL is a multi-disciplinary open access archive for the deposit and dissemination of scientific research documents, whether they are published or not. The documents may come from teaching and research institutions in France or abroad, or from public or private research centers.
L'archive ouverte pluridisciplinaire HAL, est destinée au dépôt et à la diffusion de documents scientifiques de niveau recherche, publiés ou non, émanant des établissements d'enseignement et de recherche français ou étrangers, des laboratoires publics ou privés. 


\title{
L'olfaction dans les troubles dépressifs : intérêts et perspectives
}

\section{Olfaction in depressive disorders: Issues and perspectives}

\author{
G. Brand ${ }^{\mathrm{a}, *, \mathrm{~b}}$, B. Schaal ${ }^{\mathrm{b}}$ \\ a Département neurosciences, université de Bourgogne-Franche-Comté, place Leclerc, 25000 Besançon, France \\ ${ }^{\mathrm{b}}$ Centre des sciences du goût et de l'alimentation, CNRS, laboratoire d'éthologie développementale et de psychologie cognitive, université de \\ Bourgogne-Franche-Comté (UMR 6265), 9E, boulevard Jeanne-d'Arc, 21000 Dijon, France
}

Il existe une abondante littérature, depuis plusieurs décennies, sur les liens entre olfaction et dépression. La revue de la littérature proposée ici n'a donc pas vocation à être exhaustive sur les travaux publiés mais vise plutôt à mettre en exergue les études les plus récentes et leurs apports à la compréhension des mécanismes olfactifs dans la dépression. En effet, étant donné l'existence de connexions étroites entre voies olfactives et aires cérébrales impliquées dans la régulation de l'humeur et des émotions (notamment au niveau du système limbique et des aires préfrontales), l'olfaction constitue une voie de recherche intéressante et novatrice à de nombreux égards. En premier lieu, l'étude des troubles olfactifs occurrents dans la dépression peut aider au diagnostic et surtout à la compréhension des mécanismes sous-jacents aux troubles thymiques. Les travaux publiés révèlent que l'épisode dépressif caractérisé est associé à une réduction de la sensibilité olfactive, ce qui n'est pas retrouvé dans la dépression bipolaire et la dépression saisonnière. En second lieu, il a été montré que des déficits de perception des odeurs pouvaient être à l'origine de symptômes dépressifs. Les corrélats neuro-anatomiques et neurochimiques plaident assez clairement pour un effet causal de la perte olfactive sur les troubles de l'humeur en général et, dans ce contexte, un modèle animal (rat bulbectomisé) conforte l'hypothèse du rôle non négligeable de l'olfaction dans les troubles dépressifs. En troisième lieu, plusieurs travaux tendent à prouver que les odeurs peuvent potentiellement avoir un impact sur l'amélioration des états dépressifs. Une remédiation par l'utilisation d'odeurs dans les troubles dépressifs et anxieux est une voie de recherche prometteuse, notamment du fait de l'impact sur le fonctionnement neurochimique de la dépression qui semble démontré chez l'animal.

Research on sensorial interactions with psychiatric diseases and particularly with the depressive syndrome has mainly focused on visual or auditory processes and much less on olfaction. The depressive illness is one of the most frequent psychiatric diagnoses in the community, with approximately one in five women and one in eight men experiencing a major depressive episode during their lifetime. Although genetic, epigenetic, neuroanatomical, neurochemical, neuroendocrinological and neuroimmunological changes can be detected during depression, the etiology of depression remains partly unclear. The current explanatory models are based on two main factors, i.e. pharmacological dysfunctions and stress effects. In this way and because of strong connections between olfactory pathways and cerebral areas implied in mood regulation and emotions (i.e. the limbic system and prefrontal areas), the interactions between olfaction and depression could constitute a relevant way of research at three different levels. First, olfactory dysfunction observed in depression could serve the diagnosis and contribute to a better understanding of mechanisms implied in thymic pathologies. Published papers show a decrease of olfactory sensitivity in major depression which does not occur in bipolar or saisonal depression. Second, it has been shown that olfactory deficits could induce depressive symptoms. In this context, an animal model (olfactory bulbectomized rat) reinforces the hypothesis of the important role of olfaction in
Mots clés :

Olfaction

Épisode dépressif caractérisé

Trouble bipolaire

Sensibilité olfactive

Diagnostic
Keywords:

Olfaction

Major depression Bipolar disorder Olfactory sensitivity Diagnosis

\footnotetext{
* Auteur correspondant.

Adresses e-mail : gerard.brand@univ-fcomte.fr (G. Brand), benoist.schaal@u-bourgogne.fr (B. Schaal).
} 
depression based on neuroanatomical and neurochemical observations. Third, several publications have demonstrated that odors can positively impact the depressive mood. Thus, a remediation by odors in depression appears to be a promising way. From several decades, the olfaction/depression interactions have been covered by a broad literature. Thus, the present review will not propose an exhaustive examination but aims to point out the most recently published papers and highlight their contributions to the understanding of olfactory processes implied in depression.

\section{Introduction}

Beaucoup d'études se sont focalisées sur les interactions entre sensorialité et dépression qu'il s'agisse des effets de la dépression sur la perception sensorielle [1] ou qu'il s'agisse de la modulation de l'humeur par des stimulations visuelles, auditives, voire somesthésiques. . . La dépression est l'une des affections psychiatriques les plus répandues et bien que de nombreux aspects, génétiques, épigénétiques, neuro-anatomiques, neurochimiques, neuro-endocrinologiques, neuro-immunologiques puissent être reliés à un épisode dépressif caractérisé, l'étiologie de la dépression reste encore en partie obscure [2]. Les principaux modèles explicatifs reposent sur deux facteurs principaux, les aspects pharmacologiques et les facteurs de stress. Dans ce cadre, et du fait des connexions étroites entre voies olfactives et aires cérébrales impliquées dans la régulation de l'humeur et des émotions (notamment au niveau du système limbique et des aires préfrontales), l'olfaction constitue une voie de recherche très intéressante à trois niveaux qui seront développés ci-après. En premier lieu, l'étude des troubles olfactifs occurrents dans la dépression peut aider au diagnostic et surtout à la compréhension des mécanismes sous-jacents aux troubles thymiques. En second lieu, il a été montré que des déficits de perception des odeurs pouvaient être à l'origine de symptômes dépressifs et, dans ce contexte, un modèle animal (rat bulbectomisé) conforte l'hypothèse du rôle non négligeable de l'olfaction dans les troubles dépressifs. En troisième lieu, plusieurs travaux tendent à prouver que les odeurs peuvent potentiellement avoir un impact sur l'amélioration des états dépressifs.

Il existe une abondante littérature, depuis plusieurs décennies, sur les liens entre olfaction et dépression. Cette revue de la littérature n'a donc pas vocation à être exhaustive sur les travaux publiés mais vise plutôt à mettre en exergue les études les plus récentes et leurs apports à la compréhension des mécanismes olfactifs dans la dépression.

\section{Les troubles olfactifs occurrents dans la dépression}

Dans cette première partie, les résultats des études sur la perception des odeurs au cours de la dépression seront présentés, incluant l'épisode dépressif caractérisé et la dépression bipolaire. La perception des odeurs dans les troubles anxieux, trouble obsessionnel compulsif (TOC), stress post-traumatique (SPT) et trouble panique (TP) sera également abordée. L'ensemble de ces études révèlent que l'épisode dépressif caractérisé est associé à une réduction de la sensibilité olfactive. En revanche, les scores d'identification et de discrimination ne semblent pas affectés par la maladie dépressive. La baisse de la sensibilité olfactive pourrait être due à une moindre capacité à encoder l'information olfactive et une réduction du volume des bulbes olfactifs. En revanche, ces éléments ne sont retrouvés dans la dépression bipolaire ou la dépression saisonnière.

\subsection{La mesure olfactive}

La perception des odeurs est évaluée avec des méthodes psychophysiques (sensibilité, identification, discrimination) et la mesure de paramètres tels que l'intensité de l'odeur, la valence hédonique ou la familiarité. Pour une revue complète des différents tests utilisés et commercialisés, le lecteur peut se référer aux publications suivantes [3-5]. D’autres études s'intéressent aux réactions affectives vis-à-vis des odorants avec des corrélations physiologiques et neuro-anatomiques de la perception des odeurs dans la dépression. Il est d'usage de considérer deux niveaux dans l'évaluation olfactive, le niveau dit périphérique qui concerne principalement la sensibilité aux odeurs, mesurée par les seuils de détection et le niveau dit central qui concerne l'identification, la mémorisation, la familiarité, l'estimation de l'intensité et de l'hédonicité. Cette distinction est importante au regard des zones du système olfactif impliquées :

- au niveau périphérique, il s'agit essentiellement du neuroépithélium situé dans la partie supérieure des cavités nasales et des bulbes olfactifs qui constituent le premier relais dans la remontée des informations ;

- au niveau central, il s'agit du grand nombre d'aires impliquées dans le traitement de l'information olfactive, et notamment parmi elles, l'hippocampe, l'amygdale et le cortex orbito-frontal.

\subsection{Les investigations au niveau périphérique}

Concernant l'épisode dépressif caractérisé, de très nombreuses études ont évalué la sensibilité olfactive avec des résultats controversés. En effet, les seuils de détection sont parfois augmentés, ce qui signifie que les performances sont moins bonnes chez les patients dépressifs par rapport aux sujets témoins [6,7] ou inchangés [8], une étude montrant même une amélioration des performances chez les patients dépressifs pour l'odeur d'acétate d'isoamyle [9]. En outre, certains auteurs trouvent une corrélation entre la mesure de seuil olfactif et les mesures cliniques de la dépression, y compris chez les sujets sains avec la Beck Depression Inventory (BDI) [10] tandis que d'autres n'en trouvent pas [9]. Une grande part de ces divergences peut sans doute être attribuée aux différences méthodologiques, en fonction du type de tests utilisés, des odeurs choisies ${ }^{1}$, du mode de présentation, du moment ou de la durée de la passation et bien évidemment des sujets testés eux-mêmes, en termes d'âge, de genre, de type de traitement, de durée de la prise en charge, de la comorbidité éventuelle et des différences cliniques interindividuelles. Le point le plus important est sans doute de distinguer les patients en phase de dépression aiguë et les patients en rémission. En effet, les études publiées qui évaluent plusieurs paramètres olfactifs à différents stades de la maladie montrent effectivement des différences de sensibilité olfactive dépendantes de la phase clinique $[6,7,9]$.

\footnotetext{
${ }^{1}$ La plupart des études évaluent le seuil olfactif à l'aide d'un unique odorant. Le problème est que cette sensibilité spécifique à l'odorant choisi ne reflète en rien la sensibilité générale qui s'avère extrêmement variée au regard de la multitude des odeurs potentiellement perceptibles, pouvant aller jusqu'à l'anosmie complète pour certaines.
} 
Concernant la dépression bipolaire, les travaux disponibles ne mettent pas en évidence de différence d'acuité olfactive entre patients et sujets témoins. Il semble cependant que les patients bipolaires présentant un événement déclencheur connu ont des seuils plus bas (donc de meilleures performances) que les patients n'en présentant pas, mais cette étude n'inclut pas de sujets témoins [8].

En conclusion et d'un point de vue méthodologique, l'évaluation de la sensibilité olfactive dans les troubles de l'humeur devrait toujours s'accompagner de mesures des performances cognitives et des capacités perceptives en général. En effet, des études présentées ci-dessus, il ressort que les modifications des performances olfactives sont " disorder-modality-test " spécifiques, autrement dit, extrêmement dépendantes des caractéristiques de la pathologie, des odeurs et des tests utilisés. Ces approches congruentes - capacités olfactives et cognitives - pourraient alors présenter un grand intérêt dans le diagnostic différentiel des troubles de l'humeur.

\subsection{Les investigations au niveau central}

La plupart des études montrent de façon générale que les performances d'identification des odeurs ne sont pas affectées dans le cas des troubles de l'humeur $[7,8]$. Toutefois, certaines études récentes notent qu'une diminution des performances d'identification peut être relevée dans l'épisode dépressif caractérisé par rapport à des sujets témoins [11-13] et dans la dépression bipolaire [14]. Les capacités de discrimination entre odeurs et les capacités d'évaluation de l'intensité ne varient pas entre sujets témoins et patients en épisode dépressif caractérisé [7,11,12,15]. Cependant, il pourrait exister un effet de la valence hédonique [11] avec des performances d'évaluation de l'intensité chez les patients dépressifs moins bonnes pour les odeurs plaisantes et meilleures pour les odeurs désagréables que chez les sujets témoins.

Du point de vue hédonique, les différences d'évaluation entre sujets dépressifs et sujets non dépressifs sont difficiles à établir $[8,12]$. Il existe toutefois un certain nombre d'indicateurs subtils mais pertinents au regard des liens existants entre dépression et émotion. De façon surprenante, une étude [6] note une surévaluation du caractère agréable des odeurs chez des patients dépressifs alors même que les sujets dépressifs ont généralement tendance à juger négativement les expériences émotionnelles. Dans une étude plus ancienne, Pause et al. (2001) [15] observaient une différence d'évaluation de l'hédonicité, mais uniquement pour une odeur (le citral) parmi 10 proposées. Les auteurs expliquaient cette observation par le fait que l'odeur de citral est reconnue pour ses propriétés relaxantes. Il a aussi été montré [11] que les patients en épisode dépressif caractérisé percevaient les odeurs déplaisantes comme étant plus déplaisantes et [16] percevaient les odeurs plaisantes comme moins plaisantes que les sujets témoins, suggérant ainsi une alliesthésie olfactive négative. Swiecicki et al. [8] comparent des patients dépressifs unipolaires et bipolaires et ne trouvent pas de différence d'estimation hédonique entre les deux groupes. Concernant la dépression bipolaire, Cumming et al. [14] reportent que les patients évaluent les odeurs comme étant plus plaisantes que les sujets témoins.

Il est intéressant de noter que la plupart des études consacrées à l'hédonicité dans la gustation chez les patients dépressifs sont convergentes avec les études en olfaction, à savoir peu d'effet tangible de la maladie sur l'estimation hédonique. Cette convergence entre olfaction et gustation est importante car elle suggère qu'il n'existe pas de déficit patent des circuits neuronaux impliqués dans l'évaluation des sensations agréables/désagréables en réponse à des stimulations chimiques et l'image clinique de l'anhédonie ne s'exprimerait donc pas au niveau de la perception chémosensorielle. D'un point de vue fonctionnel, cette observation est cruciale en ce qu'elle rejoint l'hypothèse que l'anhédonie
- observée avec d'autres types de stimuli, telles que des photographies de l'International Affective Picture System (IAPS), par exemple - renvoie à des dysfonctionnements motivationnels ou cognitifs perturbant l'appréciation hédonique plutôt qu'à des dysfonctionnements perceptifs.

\subsection{Les études en neuroimagerie et en neurophysiologie}

Pause et al. [17] ont montré que dans le cas d'un épisode dépressif caractérisé, les patients en début de prise en charge présentaient des réponses aux odeurs avec des amplitudes P2 et P3-1 réduites. Après traitement, les potentiels évoqués olfactifs ne varient pas entre témoins et patients dépressifs. Dans l'étude de Krüger et al. [18], les potentiels évoqués olfactifs permettent de distinguer au sein d'une population de dépressifs bipolaires ceux pour lesquels un événement déclencheur est connu de ceux pour lesquels aucun événement déclencheur n'est répertorié. Les données en neuroimagerie [7] révèlent que les bulbes olfactifs des patients dépressifs présentent des volumes moindres que les sujets témoins et qu'il existe une corrélation entre le volume et l'acuité olfactive ainsi qu'avec les scores de dépression (voir paragraphes précédents). Les auteurs posent l'hypothèse que cette diminution du volume associée à une baisse des performances olfactives pourrait être associée à une réduction de la neurogenèse au niveau du système olfactif. Cette question sera discutée en seconde partie.

\subsection{Olfaction et troubles anxieux}

La littérature relative à l'olfaction dans les troubles anxieux est rare. Les observations concernent majoritairement les troubles obsessionnels compulsifs (TOC), le stress post-traumatique (SPT) et à un degré moindre les troubles paniques (TP). Il s'agit principalement d'investigations patho-physiologiques des aires impliquées dans ces troubles, à savoir le cortex orbito-frontal, l'amygdale, l'hippocampe ou encore l'insula $[19,20]$. Au niveau périphérique, seul le TOC a été étudié du point de vue des seuils olfactifs et aucune diminution de l'acuité n'est décelée [9,21]. Au niveau central, la première publication recensée [22] trouve un déficit d'identification des odeurs chez des femmes présentant un haut niveau obsessionnel (sans toutefois qu'il s'agisse d'un TOC) comparativement à des sujets témoins. Ce déficit d'identification est confirmé [23] au sein d'un groupe de patients présentant un TOC avéré cliniquement. De façon intéressante, ce déficit d'identification est associé à des déficits de la mémoire spatiale. Dans la mesure où ces deux processus sont principalement gérés par le cortex orbito-frontal, cette observation a permis de poser l'hypothèse d'un dysfonctionnement majeur de cette aire d'intégration dans les troubles obsessionnels compulsifs. Le déficit d'identification des odeurs est également retrouvé chez des sujets victimes d'un stress post-traumatique [24], ce déficit pouvant même être un signe prédictif d'impulsivité non contrôlée voire d'agression ${ }^{2}$.

\subsection{Olfaction chez des sujets sains avec humeur dépressive}

Il est possible que les performances olfactives soient également altérées chez des personnes saines présentant une humeur dépressive modérée ou certains symptômes dépressifs sans que cela s'avère cliniquement pathologique. Ce pourrait être le cas pour la baisse de sensibilité $[10,25]$ avec des corrélats

\footnotetext{
2 Ces études concernaient en partie des vétérans victimes de SPT. Contrairement aux sujets contrôles, ils jugeaient l'odeur de diesel (liée à la mémoire traumatique des combats) comme beaucoup plus déplaisante et l'exposition à cette odeur augmentait chez eux significativement les symptômes du SPT et l'anxiété.
} 
électrophysiologiques [26] mais ne concernerait pas les performances d'identification [27].

\section{La dépression consécutive à des troubles olfactifs}

\subsection{Olfaction et qualité de vie}

Pour les sujets normosmiques, il est difficile d'imaginer l'impact sur la qualité de vie que peut avoir une perte de l'odorat. Le rôle général de l'olfaction est de nous renseigner d'une part contre les risques potentiels liés aux émanations toxiques ou aux produits alimentaires avariés et d'autre part de nous orienter vers des odeurs connotées positivement, qu'il s'agisse de l'alimentation, d'odeurs corporelles ou de substances aromatiques diverses. Les préférences (ou la valence hédonique des odeurs) sont largement déterminées par l'expérience individuelle car les tentatives explicatives par rapport à la structure moléculaire des odorants ou à une activation neurale spécifique $[28,29]$ ne sont pour l'instant pas très convaincantes.

Dans l'évaluation de la qualité de vie, la prise alimentaire et la relation aux autres font partie des critères majeurs. Dans le cas de la prise alimentaire, les odeurs jouent un rôle crucial dans le choix, l'appréciation et le rassasiement. Les molécules odorantes parviennent aux récepteurs olfactifs par deux voies distinctes, une voie directe (dite orthonasale) avant la mise en bouche et une voie indirecte (dite rétronasale) lors de la mastication. Dans ce dernier cas, les molécules volatiles empruntent alors le canal palatin pour activer les récepteurs olfactifs dans la cavité nasale. Une perte ou une diminution de l'odorat peut entraîner une perte du plaisir liée à l'alimentation et des troubles au niveau nutritionnel pouvant affecter l'état de santé des sujets concernés. En ce qui concerne la communication sociale, les odeurs jouent un rôle crucial dans les comportements reproducteurs, les comportements parentaux, l'évitement ou le choix de partenaires, le repérage des prédateurs potentiels. . . Depuis peu, il a même été démontré un rôle des odeurs dans la contagion émotionnelle comme dans le cas de l'anxiété [30] ou encore la présence de signaux chimiques dans les larmes de femmes qui provoquent une diminution de l'attractivité sexuelle et du niveau de testostérone chez les hommes [31]. Ainsi, une perte ou une diminution de l'odorat peut entraîner des perturbations dans la communication et la relation aux autres.

\subsection{Les troubles de l'odorat}

La prévalence des troubles de l'odorat dans la population générale est difficile à évaluer [32], notamment par ce qu'il existe une diversité et des niveaux d'atteintes très différents et enfin parce que les moyens pour évaluer ces troubles sont peu développés, contrairement à d'autres troubles sensoriels tels que ceux qui touchent la vision ou l'audition. En outre, beaucoup de personnes ignorent également qu'elles ont un odorat déficient [33]. Dans l'odorat, il faut distinguer les troubles quantitatifs - anosmie (perte de l'odorat, il peut s'agir d'anosmie totale ou d'anosmie partielle), hyposmie (diminution de la sensibilité aux odorants ou à certains types d'odorants), hyperosmie (élévation de la sensibilité aux odorants ou à certains types d'odorants) - mesurables à l'aide de tests et les troubles qualitatifs tels que les parosmies (déformation de la perception des odorants) ou les fantosmies (perception d'odeurs sans stimulus odorant).

L'origine post-infectieuse, notamment au niveau de la région rhinosinusienne est sans doute la plus fréquente. En effet, au cours de la vie, chacun peut souffrir à de nombreuses reprises d'infections des voies respiratoires supérieures, entraînant des obstructions nasales qui altèrent l'odorat de façon transitoire. Les traumatismes crâniens ou faciaux constituent une autre origine possible de troubles plus ou moins sévères de l'odorat. Des chocs, même mineurs peuvent provoquer des cisaillements d'axones et/ou des lésions à différents endroits des voies olfactives, qu'il s'agisse des bulbes olfactifs, des aires situées en zone temporale ou du cortex orbito-frontal. Une autre origine, moins fréquente mais irréversible est congénitale. Le trouble est principalement lié à une hypoplasie ou aplasie des bulbes olfactifs comme dans le cas du syndrome de Kallmann, associé à un hypogonadisme. Récemment, des anosmies associées au syndrome CHARGE et au syndrome de Bardet-Biedl ont été rapportées. Par ailleurs, les troubles de l'odorat d'origine neurodégénérative sont désormais bien identifiés, notamment dans la maladie d'Alzheimer et la maladie de Parkinson, ces troubles pouvant se manifester avant même l'établissement clinique de la maladie. Enfin, il reste un pourcentage non négligeable (15 à $20 \%$ ) de troubles idiopathiques de l'odorat pour lesquels les causes non identifiées pourraient relever d'effets médicamenteux, d'atteintes toxicologiques ou de dérégulation métabolique.

\subsection{Troubles de l'odorat et qualité de vie}

Les conséquences des troubles olfactifs sur la qualité de vie sont extrêmement variées comme le rapporte le recensement publié d'un millier de cas [34]. Le problème est qu'ils surviennent fréquemment dans le cas de comorbidité et qu'il est alors difficile de distinguer ce qui relève strictement des troubles de l'odorat et des troubles associés. Les principales conséquences relevées concernent la prise alimentaire avec une réduction de l'appétit, la sécurité vis-à-vis d'aliments avariés ou de produits toxiques et l'hygiène personnelle avec l'impossibilité d'évaluer sa propre odeur corporelle.

Il est légitime de penser que les répercussions sur la qualité de vie dues aux troubles de l'odorat aient secondairement un impact dépresseur. Le mécanisme le plus communément admis est que la réduction des entrées olfactives ait un impact sur le fonctionnement du système limbique. Cette hypothèse est vérifiée chez les rongeurs et chez l'homme. D'un point de vue sémiologique, dans l'étude ancienne de [35], entre un quart et un tiers des sujets qui présentent des troubles olfactifs présentent conjointement des symptômes dépressifs avec des scores à la Beck Depression Inventory (BDI) qui s'échelonnent de modéré à sévère. En particulier, les patients qui présentent des parosmies et/ou des fantosmies ont des scores de dépression élevés. D’autres études plus récentes $[36,37]$ montrent un retentissement sur la qualité de vie accompagné à la fois d'une non-acceptation de l'état de déficience et d'une résignation devant l'impossibilité d'espérer une amélioration. Cette conjonction pourrait être à l'origine des scores de dépression (de modéré à sévère) généralement relevés chez les patients présentant une absence du sens de l'odorat depuis la naissance [38].

\subsection{Neurophysiopathologie}

L'implication des troubles olfactifs dans l'étiologie de la dépression est avérée par des travaux chez l'animal. En effet, un modèle de dépression a été développé depuis une trentaine d'années chez le rat, il s'agit de la bulbectomie olfactive (OBX : olfactory bulbectomy). L'OBX est caractérisée par une destruction bilatérale des bulbes olfactifs qui induit des changements comportementaux, neurochimiques et neuro-endocrinologiques semblables à ceux observés chez les patients dépressifs $[39,40]$. L'OBX conduit à de sévères modifications dans plusieurs aires cérébrales du fait de la disparition des connexions entre les bulbes olfactifs et d'autres régions, notamment le système limbique [39-41], telle que la diminution du volume des hippocampes, également observée chez les patients dépressifs $[42,43]$. Dans le même temps, l'OBX entraîne des modifications neurochimiques également similaires à celles observées chez les patients dépressifs [44,45], 
notamment au niveau de la dopamine [46] et de la sérotonine [44]. Enfin, de nombreuses modifications comportementales telles que l'augmentation de l'hyperactivité dans un environnement inconnu ou l'anhédonie [47] sont également observées. Toutefois, les mécanismes exacts par lesquels l'OBX induit des états dépressifs via les interactions (devenues déficitaires) entre bulbes olfactifs et différentes aires cérébrales restent très mal compris. Dans ce cadre, une publication récente [48] montre le rôle probablement crucial joué par l'habenula. Les noyaux de l'habenula sont en effet impliqués dans la régulation des comportements psychomoteurs et psychosociaux sous l'influence majeure d'inputs en provenance des bulbes olfactifs. En outre, le rôle de l'habenula latérale dans la dépression est actuellement au centre des recherches causales de la dépression $[49,50]$. Les projections en partance des noyaux de l'habenula sont majoritairement dirigées vers des aires connues pour être impliquées dans l'étiologie de la dépression. L'étude histopathologique conduite chez le rat par Oral et al. [48] montre que l'OBX induit par apoptose neuronale une dégénérescence structurale de l'habenula conduisant à l'apparition des principaux symptômes de la dépression.

\subsection{Chez la personne âgée}

La question de l'humeur dépressive consécutive à une perte olfactive est particulièrement prégnante chez la personne âgée. La diminution de l'acuité olfactive avec l'âge entraîne naturellement une baisse de la perception de la qualité de vie. L'effet principal est sans conteste un retentissement au niveau de la prise alimentaire. En effet, les données de la littérature [51] relient la perte olfactive à une diminution de l'appétit et de l'appréciation des aliments, des changements dans les préférences alimentaires qui se traduisent par une restriction de la variété et une orientation vers des produits pauvres du point de vue nutritionnel. Tout cela contribue souvent à engendrer une perte de poids et une augmentation des risques de survenue de certaines maladies chroniques. Depuis longtemps [52], il a été démontré que le rassasiement sensoriel spécifique était moins important chez les personnes âgées que chez les jeunes adultes et que c'était l'un des facteurs majeurs des modifications nutritionnelles liées à l'âge. En institution, chez les personnes âgées relativement dépendantes, l'activité liée à la prise alimentaire reste l'une des sources de plaisir récurrente qui, si elle est altérée peut logiquement conduire à l'anhédonie. Peu de travaux se sont centrés sur l'étude des liens entre olfaction et dépression chez la personne âgée. Dans le grand âge, Boesveldt et al. [53] rapportent une corrélation entre fonction olfactive et dépression tout comme Seo et al. [54] qui montrent que les déficits olfactifs sont corrélés à un faible score à l'échelle de qualité de vie. Se pose toutefois la question du rôle des déficits cognitifs potentiels dans ces études dans la mesure où ils pourraient être vecteur - au moins en partie - de la baisse des performances olfactives de la personne âgée.

\section{Le rôle des odeurs dans l'amélioration de la dépression}

\subsection{Les effets de l'exposition aux odeurs}

L'exposition aux odeurs peut avoir de multiples influences et ce, à différents niveaux, physiologique, comportemental, cognitif et émotionnel. Pour ne citer que quelques exemples, les odeurs peuvent modifier la vigilance [55], l'attention [56], la perception de la douleur [57]. En outre, les odeurs peuvent avoir un impact au niveau cognitif sur des tâches simples telles que le temps de réaction [58] ou l'effet Stroop [59] mais également sur des tâches complexes comme les processus mnésiques [60]. Elles peuvent aussi avoir un impact sur le niveau d'anxiété [61]. De façon intéressante dans le contexte des troubles de l'humeur, il a été démontré depuis longtemps qu'un conditionnement par l'odeur était effectif chez l'animal et chez l'homme. La première étude d'un tel processus chez l'homme [62] couplait une odeur (présente mais non perçue par les sujets) avec une tâche inductrice d'anxiété. Quand plus tard les sujets se retrouvaient en présence de l'odeur initiale seule, et bien qu'elle ne soit pas consciemment perçue, leur niveau d'anxiété augmentait. Une étude plus récente a montré que le conditionnement olfactif pouvait être utilisé pour augmenter positivement les performances [63]. Se pose toutefois la question des mécanismes fonctionnels liés à de tels effets. Les liens neuro-anatomiques et neurochimiques entre le système olfactif et la régulation émotionnelle sont connus et plusieurs études mettent en lumière l'effet potentiellement bénéfique de certaines odeurs dans les états dépressifs, ou plus précisément sur l'humeur. Les deux hypothèses principales classiquement retenues sont un effet pharmacologique et un effet psychologique, ce qui rejoint finalement les deux types principaux de prise en charge de la dépression [64].

\subsection{Les principales explications fonctionnelles}

L'effet pharmacologique induit par les odeurs repose sur le fait que les molécules volatiles qui pénètrent dans la cavité nasale peuvent entrer dans la circulation sanguine au niveau de la muqueuse nasale et avoir ainsi un impact sur l'activité neuronale du système nerveux autonome et du système nerveux central aussi bien que sur l'activité neuroendocrinienne. Grâce à une application transdermale [65], il a été établi qu'une période de l'ordre de 20 minutes est nécessaire pour que les molécules intégrées à la circulation sanguine passent la barrière hémato-encéphalique et puissent avoir un rôle effectif au niveau central. Toutefois, la quantité de composés actifs entrant dans la circulation sanguine par inhalation est relativement faible - autrement dit à l'état de traces - comparativement à d'autres modes d'entrée comme dans le cas de l'ingestion par exemple. En outre, il a été montré depuis longtemps chez l'animal [66] qu'une exposition de longue durée induisant une inhalation prolongée, était nécessaire pour que des traces de molécules odorantes soient retrouvées dans la circulation sanguine.

L'effet psychologique suggère que les odorants puissent avoir des effets sur des paramètres tels que l'attention ou l'humeur mais les corrélats physiologiques sont difficiles à obtenir. Par exemple, alors que l'odeur de lavande est fréquemment considérée comme ayant des effets relaxants et positifs sur l'humeur, les effets physiologiquement corrélés ne sont pas retrouvés [67] ou peut-être seulement secondairement comme dans l'hypothèse d'une amélioration du sommeil améliorant alors de façon indirecte l'humeur [68].

Dans l'optique d'améliorer l'humeur, des odorants jugés universellement plaisants comme la vanille, sont habituellement utilisés [69]. Toutefois, les effets semblent dépendre du type d'odeurs, de leurs caractéristiques en termes d'hédonicité, d'intensité ou de propension à stimuler conjointement le système trigéminal (picotement, irritation, sensations de chaleur ou de fraîcheur) [70]. Ainsi, la vanille (odeur plaisante) améliore l'humeur tandis que le sulfure d'hydrogène (odeur déplaisante) produit l'effet inverse [71]. De même, l'humeur mesurée par l'échelle Positive and Negative Affect Schedule (PANAS) est significativement améliorée par l'odeur de citron et non par l'odeur de lavande [72].

Une étude récente [73] confirme que chez des modèles de rats dépressifs (soumis à des conditions de stress chronique), l'exposition à l'odeur de vanille induit une amélioration de l'état dépressif au niveau comportemental, associée à une modification des concentrations en neurotransmetteurs et notamment une élévation des taux de dopamine et de sérotonine. En revanche, dans le modèle de rats rendus dépressifs suite à une bulbectomie (OBX) et 
pour lesquels aucune amélioration n'est observée après 6 semaines sans traitement [74], l'exposition à l'odeur de vanille ne produit pas d'effets thérapeutiques [73]. Cette observation est extrêmement importante car elle suggère que l'intégrité du système olfactif est nécessaire pour un effet thérapeutique et que l'action des molécules via la circulation sanguine est de fait inopérante ou pour le moins insuffisante. Cette observation est cruciale car elle pose conjointement l'hypothèse d'un possible double mécanisme, qui additionnerait l'effet pharmacologique (via la circulation sanguine) et psychologique via le système olfactif. Cette complexité des interactions entre olfaction et dépression est bien illustrée au niveau du possible fonctionnement neurochimique présenté ci-après.

\subsection{Au niveau neurochimique}

De nombreuses études chez l'animal et chez l'homme ont montré que certaines odeurs telles que la rose, l'orange, le citron, la lavande ou encore la vanille avaient des propriétés relaxantes et apaisantes [61,75-77]. L'un des mécanismes fondamentaux par lequel l'organisme réagit au stress est l'activation de l'axe hypothalamo-hypophysaire et l'étude de Xu et al. [73] suggère que l'odeur de vanille pourrait agir à ce niveau puisque la concentration de corticostérone est différente entre les animaux soumis à un stress chronique sans et avec exposition à cette odeur. En outre, l'activation de l'amygdale et de la voie mésocorticolimbique par les odeurs pourrait être à l'origine de l'élévation du taux des neurotransmetteurs monoaminergiques dont l'effet clinique sur la dépression est prouvé [78]. Cet effet sur les concentrations en dopamine et en sérotonine a d'ailleurs déjà été montré avec l'odeur de citron [79], au niveau du cortex préfrontal et du striatum notamment. Il faut noter que dans ces études, il est tenu compte de l'adaptation aux odeurs - qui survient lors d'une exposition continue et susceptible d'empêcher l'effet thérapeutique - par la mise en place de protocoles d'expositions répétées et suffisamment espacées les unes des autres dans le temps. De même, l'intensité de l'odorant est calibrée de sorte à n'être ni trop faible et donc peu ou pas perçue, ni trop forte et donc désagréable ou trop irritante. Les questions méthodologiques liées à l'utilisation des odeurs restent donc nombreuses et les recherches futures doivent proposer des protocoles relativement standardisés pour fournir des résultats comparables en termes d'effet thérapeutique.

\section{Conclusion}

En premier lieu, une revue de la littérature portant sur les relations causales et fonctionnelles entre olfaction et dépression révèle que l'épisode dépressif caractérisé est associé à une réduction de la sensibilité olfactive. En revanche, les scores d'identification, de discrimination, d'estimation hédonique et de l'intensité ne semblent pas (ou peu) affectés par la maladie dépressive. La baisse de la sensibilité olfactive pourrait être due à une moindre capacité à encoder l'information olfactive, liée principalement à une perte fonctionnelle au niveau des bulbes olfactifs. En revanche, ces éléments ne sont pas retrouvés de façon convaincante dans la dépression bipolaire et la dépression saisonnière. Toutefois, la plupart des études ne prennent pas en considération certains biais méthodologiques. En effet, dans tous les cas, il apparaîtrait nécessaire que les tâches olfactives effectuées s'accompagnent de mesures précises des performances cognitives et des capacités perceptives en général. Il ressort assez clairement que les scores aux épreuves olfactives sont dépendants du type de test et des conditions de passation, des odeurs utilisées, des caractéristiques des patients et de la prise en charge.

En second lieu, les corrélats neuro-anatomiques et neurochimiques plaident assez clairement pour un effet causal de la perte olfactive sur les troubles de l'humeur en général. Ce constat est étayé par de nombreux travaux (en particulier chez l'animal) et mérite d'être pris en considération du point de vue clinique. En effet, outre la dépression elle-même, la diminution des performances olfactives survient dans de nombreux cas tels que l'avancée en âge mais aussi dans certaines maladies neurodégénératives telles que la maladie de Parkinson ou la maladie d'Alzheimer et il est bien connu que la probabilité de comorbidité avec la dépression est forte [80].

En troisième lieu, une remédiation par l'utilisation d'odeurs est envisageable dans les troubles dépressifs et anxieux. Il s'agit d'une voie de recherche prometteuse, notamment du fait de l'impact sur le fonctionnement neurochimique de la dépression qui semble démontré chez l'animal.

\section{Déclaration de liens d'intérêts}

Les auteurs déclarent ne pas avoir de liens d'intérêts.

\section{Références}

[1] Canbeyli R. Sensorimotor modulation of mood and depression: an integrative review. Behav Brain Res 2010;207:249-64

[2] Kern N, Sheldrick AJ, Schmidt FM, et al. Neurobiology of depression and novel antidepressant drug targets. Curr Pharm Design 2012;18:5791-801.

[3] Atanasova B, Hernadez N, Van Nieuwenhuijzen P, et al. Psychophysical, neurophysiological and neurobiological investigation of olfactory process in humans. Olfactory impairment in some neuropsychiatric disorders. In: Weiss LE, Atwood JM, editors. The biology of odors: sources, olfaction and response. New York: Nova Science Publishers; 2011. p. 1-67.

[4] Doty RL. Psychophysical measurement of human olfactory function. In: Doty RL, Laing DG, editors. Handbook of olfaction and gustation. New York: John Wiley \& Sons; 2015. p. 225-60.

[5] Welge-Luessen A, Hummel T. Management of smell and taste disorders. A practical guide for clinicians. Stuttgart: Thieme; 2015

[6] Lombion-Pouthier S, Vandel P, Nezelof S, et al. Odor perception in patients with mood disorders. J Affect Disord 2006;90:187-91.

[7] Negoias S, Croy I, Gerber J, et al. Reduced olfactory bulb volume and olfactory sensitivity in patients with acute major depression. Neuroscience 2010;169:415-21.

[8] Swiecicki L, Zatorski P, Bzinkowska D, et al. Gustatory and olfactory function in patients with unipolar and bipolar depresion. Prog Neuropsychophysiol 2009;33:827-34

[9] Gross-Isseroff R, Luca-Haimovici K, Sasson Y, et al. Olfactory sensitivity in major depressive disorder and obsessive compulsive disorder. Biol Psychiatry 1994;35:798-802

[10] Pollatos O, Albrecht J, Kopietz R, et al. Reduced olfactory sensitivity in subjects with depressive symptoms. J Affect Disord 2007:102:101-8.

[11] Atanasova B, El-Hage W, Chabanet C, et al. Olfactory anhedonia and negative olfactory alliesthesia in depressed patients. Psychiatr Res 2010;176:190-6.

[12] Clepce M, Gossler A, Reich K, et al. The relation between depression, anhedonia and olfactory hedonic estimates. A pilot study in major depression. Neurosci Lett 2010;57:139-43.

[13] Zucco GM, Bollini F. Odour recognition memory and odour identification in patients with mild and severe major depressive disorders. Psychiatr Res 2011;190:217-20

[14] Cumming AG, Matthews NL, Park S. Olfactory identification and preference in bipolar disorder and schizophrenia. Eur Arch Psy Clin N 2011;261:251-9.

[15] Pause BM, Miranda A, Göder R, et al. Reduced olfactory performance in patients with major depression. J Psychiatr Res 2001;35:271-7.

[16] Naudin M, El-Hage W, Gomes M, et al. State and trait olfactory markers of major depression. PloS One 2012;7:e46938.

[17] Pause BM, Raack N, Sojka B, et al. Convergent and divergent effects of odors and emotions in depression. Psychophysiology 2003;40:209-25.

[18] Krüger S, Frasnelli J, Braünig P, et al. Increased olfactory sensitivity in euthymic patients with bipolar disorder with event-related episodes compared with patients with bipolar disorder without such episodes. J Psychiatry Neurosci 2006;31:263-70

[19] Etkin A, Wager TD. Functional neuroimaging of anxiety: a meta-analysis of emotional processing in PTSD, social anxiety disorder and specific phobia. Am J Psychiatry 2007;164:1476-88.

[20] Engel K, Bandelow B, Gruber O, et al. Neuroimaging in anxiety disorders. Neural Transm 2009:116:703-16.

[21] Hermesh H, Zohar J, Weizmann A, et al. Orbitofrontal cortex dysfunction in obsessive-compulsive disorder. Eur Neuropsychopharmacol 1999;9:415-20.

[22] Goldberg ED, Goldberg RJ, Vanoppen B. Sense of smell and obsessional behaviour. Am J Psychiatry 1991;148:1757.

[23] Barnett R, Maruff P, Purcell R, et al. Impairment of olfactory identification in obsessive-compulsive disorder. Psychol Med 1999;29:1233-77. 
[24] Dileo JF, Brewer WJ, Hopwood M, et al. Olfactory identification dysfunction, aggression and impulsivity in war veterans with posttraumatic stress disorder. Psychol Med 2007;38:523-31.

[25] Pouliot S, Bourgeat F, Barkat S, et al. Increase in anhedonia level in menopausal women is accompanied by a shift in olfactory function. Chemosens Percept $2008 ; 1: 43-7$.

[26] Laudien JH, Küster D, Sojka B, et al. Central odor processing in subjects experiencing helplessness. Brain Res 2006;1120:141-50.

[27] Scinska A, Wrobel E, Korkosz A, et al. Depressive symptoms and olfactory function in older adults. Psychiatry Clin Neurosci 2008;62:450-6.

[28] Khan RM, Luck CH, Flinker A, et al. Prediciting odor pleasantness from odorant structure: pleasantness as a reflection of the physical word. J Neurosci 2007;27:10015-23.

[29] Lapid H, Shushan S, Plotkin A, et al. Neural activity at the human olfactory epithelium reflects olfactory perception. Nat Neurosci 2011;14:1455-61.

[30] Prehn-Kristensen A, Wiesner C, Bergmann TO, et al. Induction of empathy by the smell of anxiety. PloS One 2009;4:e5987.

[31] Gelstein S, Yeshurun Y, Rozenkrantz L, et al. Human tears contain a chemosignal. Science 2011;331:226-30.

[32] Nordin S, Bramerson A. Complaints of olfactory disorders: epidemiology, assessment and clinical implications. Curr Opin Allergy Clin 2008;8:10-5.

[33] Shu CH, Lee PO, Lan MY, et al. Factors affecting the impact of olfactory loss on the quality of life and emotional coping ability. Rhinology 2011;49: 337-41.

[34] Keller A, Malaspina D. Hidden consequences of olfactory dysfunction: a patient report series. BMC ENT Dis 2013;13:8.

[35] Deems DA, Doty RL, Settle RG, et al. Smell and taste disorders, a study of 750 patients from the University of Pennsylvania Smell and Taste Center. Arch Otolaryngol 1991;117:519-28.

[36] Nordin S, Blomqvist EH, Olsson P, et al. Effects of smell loss on daily life and adopted coping strategies in patients with nasal polyposis with asthma. Acta Otolaryngol 2011;131:826-32.

[37] Smeets MA, Veldhuizen MG, Galle S, et al. Sense of smell disorder and healthrelated quality of life. Rehabil Psychol 2009;54:404-12.

[38] Croy I, Negoias S, Novakova L, et al. Learning about the functions of the olfactory system from people without a sense of smell. PloS One 2012;7:3.

[39] Kelly JP, Wrynn AS, Leonard BE. The olfactory bulbectomized rat as a model of depression: an update. Pharmacol Ther 1997;74:299-316.

[40] Song C, Leonard BE. The olfactory bulbectomized rat as a model of depression. Neurosci Biobehav Rev 2005;29:627-47.

[41] Mineur YS, Belzung C, Crusio W. Functional implications of neurogenesis impairment following chronic mild stress in mice. Neuroscience 2007;150: 251-9.

[42] Sheline YL, Gado MH, Kraemer HC. Untreated depression and hippocampal volume loss. Am J Psychiatry 2003;160:1516-8.

[43] McEwen BS, Olié P. Neurobiology of mood, anxiety and emotions as revealed by studies of unique antidepressant: tianeptine. Mol Psychiatry 2005;10: 525-37.

[44] van der Stelt HM, Breuer ME, Olivier B, et al. Permanent deficits in serotonergic functioning of olfactory bulbectomized rats: an in vivo microdialysis study. Biol Psychiatry 2005;57:1061-7.

[45] Breuer ME, Groenink L, Oosting RS, et al. Long-term behavioral changes after cessation of chronic antidepressant treatment in olfactory bulbectomized rats. Biol Psychiatry 2007;61:990-5.

[46] Masini CV, Holmes PV, Freeman KG, et al. Dopamine overflow is increased in olfactory bulbectomized rats: an in vivo microdialysis study. Physiol Behav 2004;81:111-9.

[47] Jarosik J, Legutko B, Unsicker K, et al. Antidepressant-mediated reversal of abnormal behavior and neurodegeneration in mice following olfactory bulbectomy. Exp Neurol 2007;204:20-8.

[48] Oral E, Aydin MD, Aydin N, et al. How olfaction disorders can cause depression? The role of habenular degeneration. Neuroscience 2013;240:63-9.

[49] Proulx CD, Hikosaka O, Malinow R. Reward processing by the lateral habenula in normal and depressive behaviors. Nat Neurosci 2014;17:1146-52.

[50] Zhao H, Zhang BL, Yang SJ, et al. The role of lateral habenula-dorsal raphe nucleus circuits in higher brain functions and psychiatric illness. Behav Brain Res 2015;277:89-98.

[51] Karpa MJ, Gopinath B, Rochtchina E, et al. Prevalence and neurodegenerative or other associations with olfactory impairment in an older community. J Aging Health 2010;22:154-68.

[52] Rolls BJ, McDermott TM. Effects of age on sensory-specific satiety. Am J Clin Nutr 1991;54:988-96.
[53] Boesveldt S, Lindau ST, McClintock MK, et al. Gustatory and olfactory dysfunction in older adults: a national probability study. Rhinology 2011;49:324-30.

[54] Seo HS, Jeon KJ, Hummel T, et al. Influences of olfactory impairment on depression, cognitive performance and quality of life in Korean Elderly. Eur Arch Otorhinolaryngol 2009;266:1739-45.

[55] Norrish MI, Dwyer KL. Preliminary investigation of the effect of peppermint oil on an objective measure of daytime sleepiness. Int J Psychophysiol 2005;55:291-8.

[56] Michael GA, Jacquot L, Millot JL, et al. Ambient odors influence the amplitude and time course of visual distraction. Behav Neurosci 2005;119:708-15.

[57] Villemure C, Slotnick BM, Bushnell MC. Effects of odours on pain perception: deciphering the roles of emotion and attention. Pain 2003;106:101-8.

[58] Brand G, Millot JL. Effects of ambient odors on reaction time in humans. Neurosci Lett 2002;322:79-82.

[59] Finkelmeyer A, Kellermann T, Bude D, et al. Effects of aversive odour presentation on inhibitory control in the Stroop colour-word interference task. BMC Neurosci 2010;11:131.

[60] Castellanos KM, Hudson JA, Haviland-Jones J, et al. Does exposure to ambient odors influence the emotional content of memories? Am J Psychol 2010;123:269-79.

[61] Lehrner J, Marwinski G, Lehr S, et al. Ambient odor of orange and lavender reduce anxiety and improve mood in a dental office. Physiol Behav $2005 ; 86: 92-5$.

[62] Kirk-Smith MD, Dodd GH, van Toller C. Unconscious odor conditioning in human subjects. Biol Psychol 1983;17:221-31.

[63] Chu S. Olfactory conditioning of positive performance in humans. Chem Senses 2008;33:65-71.

[64] Quidé Y, Witteveen AB, El-Hage W, et al. Differences between effects of psychological versus pharmacological treatments on functional and morphological brain alterations in anxiety disorders and major depressive disorder: a review. Neurosci Biobehav Rev 2012;36:626-44.

[65] Heuberger E, Redhammer S, Buchbauer G. Transdermal absorption of (-) linalool induces autonomic deactivation but has no impact on ratings of well-being in humans. Neuropsychopharmacol 2004;29:1925-32.

[66] Jirovitch J, Buchbauer G, Jager W, et al. Analysis of fragrance compunds in blood samples of mice by gas chromatography, mass spetrometry, GC/FTIR and GC/AES after inhalation of sandalwood oil. Biomed Chromatogr 1992;6:133-4.

[67] Motomura N, Sakurai A, Yotsuya Y. Reduction of mental stress with lavender odorant. Percept Mot Skills 2001;93:713-8.

[68] Goel N, Kim H, Lao RP. An olfactory stimulus modifies nighttime sleep in young men and women. Chronobiol Int 2005;22:889-904.

[69] Seuberg J, Rea AF, Loughead J, et al. Mood induction with olfactory stimuli reveals differential affective responses in males and females. Chem Senses 2009;34:77-84.

[70] Brand G. Olfactory/trigeminal interactions in nasal chemoreception. Neurosci Biobehav Rev 2006;30:908-17.

[71] Weber ST, Heuberger E. The impact of natural odors on affective states in humans. Chem Senses 2008;33:441-7.

[72] Kiecolt-Glaser JK, Graham JE, Malarkey WB, et al. Olfactory influences on mood andautonomic, endocrine and immune function. Psychoneuroendocrinology 2008;33:328-39

[73] Xu J, Xu H, Liu Y, et al. Vanillin-induced amelioration of depression-like behaviors in rats by modulating monoamine neurotransmitters in the brain. Psychiatr Res 2015;225:509-14.

[74] Megan EB, Lucianne G, Ronald SO, et al. Long-term behavioral changes after cessation of chronic antidepressant treatment in olfactory bulbectomized rats. Biol Psychiatry 2007;61:990-5.

[75] Almeida RN, Motta SC, Brito FC, et al. Anxiolytic like effects of rose oil inhalation on the elevated plus maze test in rats. Pharmacol Biochem Behav 2004:77:361-4.

[76] Bradley BF, Starkey NJ, Brown SI, et al. Anxiolytic effect of lavandula angustifolia odour on the mongolian gerbil elevated plus maze. J Ethnopharmacol 2007;111:517-25

[77] Matsukawa M, Imada M, Murakami T, et al. Rose odor can innately counteract predator odor. Brain Res 2011;1381:117-23.

[78] Belmaker RH, Agam G. Major depressive disorder. New Engl J Med $2008 \cdot 358 \cdot 55-68$

[79] Komiya M, Takachi T, Harada E. Lemon oil vapor causes an anti-stress effect via modulating the 5-HT and DA activities in mice. Behav Brain Res 2006;172:240-9.

[80] Naudin M, Atanasova B. Olfactory markers of depression and Alzheimer's disease. Neurosci Biobehav Rev 2014;45:262-70. 